\title{
Níveis de estresse e características sociobiográficas de alunos de pós-graduação
}

\author{
Levels of estresse and sociobiographic characteristics among graduate students \\ Niveles de estrés y características socio biográficas de alumnos de posgrado
}

\author{
Lucia Emmanoel Novaes Malagris* \\ Ana Teresa Rocco Suassuna ${ }^{* *}$ \\ Débora Ventura Bezerra ${ }^{* * *}$ \\ Heitor Pontes Hirata ${ }^{* * *}$ \\ José Luiz Fontes Monteiro ${ }^{* * * *}$ \\ Leila Rodrigues da Silva ${ }^{* * * * *}$ \\ Marília da Conceição Morais Lopes ${ }^{* * * * * *}$ \\ Tama Souza Santos ${ }^{* * * * * *}$
}

\begin{abstract}
Resumo
Buscou investigar-se a presença e o nível de estresse em alunos de pós-graduação da UFRJ, além de caracterizar a amostra. Usou-se o inventário de sintomas de estresse para adultos de Lipp e um questionário sociobiográfico. Participaram 140 alunos voluntários de todos os centros universitários. A idade média dos participantes foi de $30.3 \pm 7.5$, sendo 74 mulheres e 66 homens. Verificou-se que $58,6 \%$ dos alunos encontravamse estressados. $\mathrm{O}$ índice de estresse mais elevado foi encontrado no Centro de Ciências da Matemática e da Natureza (82,4\%), seguindose do Centro Tecnológico (61\%), Fórum de Ciência e Cultura (60\%),
\end{abstract}

Texto recebido em fevereiro de 2009 e aprovado para publicação em março de 2009.

Professora adjunta do Instituto de Psicologia, Departamento de Psicologia Clínica, Universidade Federal do Rio de Janeiro, e-mail: lucianovaes@terra.com.br.

** Diretora da Divisão de Assuntos Estudantis da Pós-graduação e Pesquisa da Universidade Federal do Rio de Janeiro UFRJ, e-mail: ateresa@pr2.ufrj.br.

${ }^{* * *}$ Graduanda do Curso de Psicologia do Instituto de Psicologia da UFRJ (Bolsa-auxílio da Pró-reitoria de Pós-graduação da UFRJ), atualmente psicóloga - UFRJ, e-mail: deboraventu@yahoo.com.br.

**** Graduando do Curso de Psicologia do Instituto de Psicologia da UFRJ (Bolsa-auxílio da Pró-reitoria de Pós-graduação da UFRJ), atualmente psicólogo - UFRJ, e-mail: heitoronline@hotmail.com.

**** Professor titular do Programa de Engenharia Química da COPPE - Universidade Federal do Rio de Janeiro. Atualmente professor titular aposentado - UFRJ, e-mail: monteiro@peq.coppe.ufrj.br.

${ }^{* * * *}$ Professora adjunta do Instituto de Filosofia e Ciências Sociais - Departamento de História da Universidade Federal do Rio de Janeiro, atualmente professora associada - UFRJ, e-mail: leila.rlk@terra.com.br.

****** Assessora técnica da Pró-reitoria de Pós-graduação e Pesquisa, atualmente substituta da superintendente administrativa da Pró-reitoria de Pós-graduação e Pesquisa - UFRJ, e-mail: marilia@pr2.ufrj.br.

${ }^{* * * * x+* *}$ Mestra em Psicologia pela Universidade Federal do Rio de Janeiro - UFRJ, e-mail: tamasantos@yahoo.com.br. 
Centro de Letras e Artes e Centro de Filosofia e Ciências Humanas (55\%), Centro de Ciências Jurídicas e Econômicas e Centro de Ciências da Saúde (50\%). O nível de estresse se mostra bastante preocupante, podendo relacionar-se a demandas específicas de um curso stricto sensu. É importante desenvolver estudos que identifiquem os eestresseores específicos.

Palavras-chave: estresse; estudantes; pós-graduação.

\section{Abstract}

The objective of this study was to investigate the estresse presence and level in graduate students from the Federal University of Rio de Janeiro and characterize the sample. We used the Lipp's Estresse Symptoms Inventory for Adults and a sociobiographic questionnaire. The 140 participants were volunteer students from all University Schools. The participants' average age was $30.3 \pm 7,5$, including 74 female and 66 male. We observed that $58.6 \%$ of the students were estresseed. The highest estresse level was observed in the School of Mathematics and Natural Sciences (82.4\%), followed by the Schools of Technology (61\%), Science and Culture (60\%), Languages and Arts and Philosophy and Humanities (55\%), Law and Economics and Health Sciences (50\%). The estresse level found in the research is extremely worrying, once it can be related to specific demands from a graduate course. It is important to develop studies in order to identify specific estresseors.

Keywords: estresse, students, graduate

\section{Resumen}

El objetivo ha sido investigar la presencia y el nivel de estrés en estudiantes de postgrado de la UFRJ, además caracterizar la muestra. Se utilizó el Inventario de Síntomas de Estrés de Adultos de Lipp y cuestionario socio biográfico. Estudiantes voluntarios, 140, han participado en todos los centros universitarios. La edad media de los participantes fue de 30 \pm 7,5, 74 mujeres y 66 hombres. Se encontró $58,6 \%$ de los estudiantes estresados. El numero más elevado de estrés se encontró en el Centro de Ciencias matemáticas y da Naturaleza (82, 4\%), seguido por el Centro Tecnológico (61\%), Foro de Ciencia y Cultura (60\%), Centro de Artes y Letras y Centro de Filosofía y Ciencias Humanas (55\%), Centro de Economía y Ciencias Jurídicas y Centro de Ciencias de la Salud (50\%). El nivel de estrés se muestra muy preocupante y puede estar relacionado con demandas específicas de un curso stricto sensu. Importante desarrollar estudios para identificación de los factores estresantes específicos.

Palabras clave: estrés, estudiantes, postgrado. 


\section{Introdução}

omo parte dos objetivos da então Divisão de Assuntos Estudantis (DAE) da Pró-reitoria de Pós-graduação e Pesquisa (PR2) da Universidade Federal do Rio de Janeiro (UFRJ), pretendeu-se desenvolver, ao longo de 2007, açōes de afirmação da universidade como instituição educacional de ensino superior que demonstrasse uma preocupação além dos aspectos estritamente relacionados à formação acadêmica de seus alunos. Partiu-se, assim, da concepção de que o processo de aprendizagem não se resume somente ao acadêmico, trazendo também, em seu bojo, os aspectos psicológicos, sociais, econômicos, culturais e de lazer como forma de atingir a excelência de ensino persistida pela UFRJ. As ações devem estar em consonância com as reais necessidades, agregando nelas o valor social.

Com a proposta de conhecer melhor o segmento da pós-graduação e pensar no estado psicológico de seus estudantes, o estresse emocional mostrou-se uma variável relevante, já que pode contribuir para prejuízos incalculáveis na qualidade de vida do estudante. Dessa forma, optou-se, na época, pela realização de um estudo que visasse a caracterizar os alunos do segmento da pós-graduação e investigar a presença e o nível de estresse deles. Este estudo pode servir de base para novos trabalhos que, somados, podem contribuir para o desenvolvimento de programas de atenção a esse grupo de estudantes, objetivando a melhoria da qualidade de vida e o rendimento acadêmico.

$\mathrm{O}$ estresse vem sendo tema de grande interesse em diversos âmbitos, o que parece decorrer das implicações biopsicossociais geradas por ele, as quais têm sido descritas por vários autores (Lipp \& Novaes, 2003; Straub, 2005). Os impactos do estresse podem ser verificados em diversos contextos, como no familiar, no social, no acadêmico/ocupacional e na área da saúde. No que se refere ao contexto acadêmico, a pós-graduação requer atenção quando se constitui em um momento de fortes pressões e cobranças para os estudantes, sendo, portanto, objeto de estudo nesta pesquisa. Cabe enfatizar que, segundo alguns autores, as atividades científicas no Brasil se desenvolvem à custa de grande desgaste emocional por parte das pessoas envolvidas (De Meis, Velloso, Lannes, Carmo \& De Meis, 2003), dentre elas, os alunos de pós-graduação.

O termo estresse, com sua concepção atual, foi uma denominação do fisiologista canadense Hans Selye (Selye, 1965). Trata-se de um estado de tensão que está relacionado a uma ruptura no equilíbrio interno do organismo e, até certo nível, é uma condição necessária e saudável que possibilita a realização de atividades do cotidiano por parte de qualquer ser humano (Lipp, 2004b; Straub, 2005). Gazzaniga e Heatherton (2005) se referem ao estresse como 
um padrão de respostas comportamentais e fisiológicas que ocorre diante de situações que excedem a capacidade de resposta e adaptação do organismo. Todos os estímulos que causam a quebra do equilíbrio das funções do corpo podem ser considerados eestresseores (Selye, 1965; Lipp \& Malagris, 1995; Lipp, 1996; Lipp \& Malagris, 2001; Straub, 2005). O processo de estresse envolve, entre outras alterações, secreção de adrenalina, podendo produzir diversas manifestações sistêmicas, como distúrbios fisiológicos e psicológicos (Margis, Picon, Cosner \& Silveira, 2003; Straub, 2005; Praag, Kloet \& Os, 2005; Malhotra \& Mehta, 2008). No entanto, ele se constitui numa tentativa de manutenção da estabilidade do organismo (Schmitt, 1999). Cabe afirmar que o estresse psicológico se constitui em um fenômeno tão primitivo como o ser humano e o acompanha por toda a história (Ahmad \& Bano, 2008).

Selye (1965) descreveu o processo de estresse em três fases: alerta, resistência e exaustão. A fase de alerta é considerada como positiva, uma vez que é o estágio no qual a ansiedade é necessária para manter o indivíduo preparado para a ação. Nesse momento, o organismo se prepara para o que Cannon (1939) denominou "luta ou fuga". Posteriormente, ocorre a fase de resistência, resultante de um acúmulo de tensão proveniente da fase anterior. O organismo passa a buscar a resistência ao desgaste sofrido previamente. Se a reserva de energia adaptativa da pessoa for suficiente, ela se recupera e sai do processo de estresse. Caso contrário, o processo pode avançar para a terceira fase, na qual ocorre uma série de sintomas que geram queda acentuada na produtividade e vulnerabilidade a vírus e bactérias (Selye, 1965; Lipp \& Malagris, 2001). Nesse caso, o indivíduo já está na fase mais negativa do estresse: a de exaustão. Esse é o momento em que, em geral, há patologia envolvida. Ocorre desequilíbrio interior de forma drástica. $\mathrm{O}$ risco de desenvolver depressão é muito grande, há falta de concentração, falhas na memória e impossibilidade de trabalhar. Impulsividade e decisóes equivocadas passam a ser comuns. Doenças graves, como úlceras, pressão alta, psoríase, vitiligo, urticária crônica e infarto agudo do miocárdio, são mais prováveis de ocorrer (Lipp, 1991; Malagris, 1996; Pinto, 1996; Lipp, 2000; Lipp \& Malagris, 2001; Csef \& Hefner, 2005; Malhotra \& Mehta, 2008). O surgimento dessas enfermidades, no entanto, não está associado somente ao estresse, mas também à sensibilidade de cada órgão e de cada sistema do corpo, podendo desencadear doenças que antes estavam latentes (Malagris \& Fiorito, 2006).

Lipp (2000) acrescentou recentemente uma fase ao modelo trifásico do estresse de Selye: a fase de quase exaustão, situada entre as fases de resistência e exaustão. Assim, a autora passou a denominar o modelo de quadrifásico do estresse (Lipp, Malagris \& Novais, 2007). Nafase de quase exaustão, a resistência 
física e emocional fica consistentemente abalada, havendo desconforto e instabilidade emocional. As defesas imunológicas ficam mais comprometidas se comparadas à fase anterior, aumentando a propensão a doenças físicas. $\mathrm{O}$ estresse excessivo pode afetar a qualidade de vida do indivíduo em cinco áreas básicas da vida: social, afetiva, profissional, espiritual e da saúde (Lipp, 2005), e podendo refletir na sociedade (Malagris, 2001).

As fontes de estresse são chamadas de eestresseores e podem se referir a qualquer estímulo, negativo ou positivo, que provoque um estado emocional forte, gerando uma quebra da homeostase interna e exigindo alguma adaptação (Lipp \& Malagris, 2001; Straub, 2005; Lipp, Malagris \& Novais, 2007). Elas podem ser classificadas em internas (Lipp, 2004, 2005), tais como pensamentos rígidos, valores antigos que não se adéquam à realidade atual e expectativas impossíveis de serem preenchidas. Existem também as fontes externas (Lipp, 2004a; Lipp, Malagris \& Novais, 2007), que podem ter o seu potencial de ação alterado para mais ou para menos, dependendo de conjugação ou não com fontes internas (Greenberger \& Padesky, 1999).

Ao refletir-se sobre as implicações macrossociais do estresse, surge a necessidade de preveni-lo e tratá-lo. Desse modo, buscar-se-á melhoria da qualidade de vida e adultos resistentes com capacidade de se adaptarem tanto a mudanças na vida pessoal quanto a mudanças que afetem a sociedade de modo geral, incluindo aquelas relacionadas ao meio científico e acadêmico.

Diante da relevância do tema, atenta-se para a necessidade de mais investigaçôes dentro do campo, a fim de discutir estratégias para lidar com os eestresseores e alternativas para enfrentar o desafio de viver com as pressóes e demandas da vida cotidiana, mantendo a saúde e administrando o estresse. No âmbito da pós-graduação, a prevenção e o controle do estresse podem ser fundamentais para influenciar positivamente o desempenho acadêmico e para que os alunos usufruam com saúde e prazer de uma etapa que pode ser enriquecedora para suas vidas.

\section{O estresse no estudante de pós-graduação}

Há tempos, existem estudos que se preocupam com a influência da formação acadêmica nasaúdedoaluno. No quediz respeitoaoensinosuperior, encontramse pesquisas mais voltadas para a graduação, como o estudo brasileiro de Calais et al. (2007), que teve como objetivo investigar calouros e veteranos do curso de Jornalismo e concluiu que $57 \%$ dos calouros e $48 \%$ dos veteranos estavam eestresseados, e que a maioria era de mulheres. Baptista e Campos (2000) investigaram o índice de estresse entre alunos do curso de Psicologia de uma universidade da cidade de São Paulo e encontraram 46\% deles eestresseados. 
Já a situação da pós-graduação é menos explorada, como enfatizam Louzada e Silva Filho (2005). Entre os estudos encontrados na literatura brasileira, pode-se citar o realizado por Santos e Alves Júnior (2007). Tal estudo buscou investigar 27 mestrandos em ciências da saúde com a faixa etária principalmente entre 30 e 39 anos e revelou que $40,7 \%$ de estudantes estavam eestresseados, $81,8 \%$ na fase de resistência, com predominância de sintomas físicos $(63,6 \%)$, sendo que a maioria dos eestresseados era composta por mulheres $(81,8 \%)$. Sabe-se que o desenvolvimento da ciência no Brasil acontece à custa de um enorme desgaste emocional das pessoas envolvidas (De Meis, Velloso, Lannes, Carmo \& De Meis, 2003). Além disso, estudos verificaram as presenças da síndrome de Burnout (De Meis, Velloso, Lannes, Carmo \& De Meis, 2003) e de crises adaptativas ou psicopatológicas (Nogueira-Martins, Fagnani Neto, Macedo, Cítero \& Mari, 2004) entre pós-graduandos brasileiros. Em nível internacional, é interessante citar estudos americanos com alunos de pós-graduação como o de Stecker (2004), que encontrou sintomas de depressão, estresse e uso de substâncias e verificou associação entre níveis de estresse e sintomas de depressão, além de baixo suporte social. Focando nesse mesmo segmento acadêmico, Hyun, Quinn, Madon e Lustig (2007) concluíram que existe uma saúde inapropriada entre pós-graduandos estrangeiros e enfatizaram a necessidade de maior atenção para a relação dos alunos com seus orientadores e com o suporte financeiro. Pfeifer, Kranz e Scoggin (2008) examinaram o estresse percebido em estudantes do sexo feminino de mestrado do curso de terapia ocupacional e concluíram que 66,4\% destes avaliaram o seu nível de estresse como acima da média. Essas estudantes relataram sentir-se sobrecarregadas e confusas em relação às expectativas do curso e desejavam mais experiência prática.

Entre os eestresseores externos que atingem essa população, encontram-se: alta competitividade, cumprimento de prazos, sobrecarga de atividades, inserção no mercado de trabalho, incertezas quanto ao futuro profissional, preocupação com o tempo médio de titulação, curso das disciplinas, situações de avaliação (seleção, exame de conhecimentos, qualificação e defesa da dissertação ou tese), relacionamento com o orientador e demais professores, procedimentos do Comitê de Ética, uso da língua inglesa, desenvolvimento da dissertação ou tese (busca de material bibliográfico, coleta e análise dos dados, redação), cobrança de produtividade (principalmente publicações), falta de autodisciplina, problemas na obtenção de bolsa ou financiamento, mudanças nas diretrizes da universidade e crescente número de orientandos por professor (Bujdoso, 2005; Duque, Brondani \& Luna, 2005; Louzada \& Silva Filho, 2005; Louzada \& Silva Filho, 2002). Byars (2005) acrescenta questóes interpessoais e preocupaçôes financeiras como eestresseores comuns em alunos de pós-graduação. 
Segundo Louzada e Silva Filho (2005), a vulnerabilidade dos pósgraduandos a experimentar estresse está relacionada às políticas educacionais e de ciência e tecnologia vigentes, às regras acadêmicas do programa de pós-graduação no qual o aluno está inserido e ao modo como ele interpreta as situações. Além disso, os autores acrescentam que o apoio recebido do orientador, da instituição ou de profissionais, é essencial no enfrentamento do estresse acadêmico (Louzada \& Silva Filho, 2002).

Cabe lembrar que a pós-graduação é uma das colunas para o desenvolvimento de uma nação. A maior parte dos mestres e doutores atua em duas áreas vitais: docência e pesquisa. Logo, deles dependerá fortemente a qualidade dos profissionais que chegarão ao mercado de trabalho no futuro, bem como será determinante a contribuição deles nos avanços tecnológicos que possibilitam o desenvolvimento de um país. Por isso, além do empenho para aperfeiçoar os aspectos acadêmicos e produtivos da pós-graduação, também é preciso investir no aprimoramento da qualidade de vida dos pós-graduandos e na sua afirmação como profissionais, pois esses são elementos estreitamente relacionados (Duque, Brondani \& Luna, 2005).

Conhecendo-se o nível de estresse dos estudantes em questão, é possível pensar na implementação de programas que visem a ajudar o estudante no controle do estresse e, ou, repensar questôes específicas e atuais do âmbito da pós-graduação. A prevenção e, ou, controle do estresse pode ser um valioso meio para evitarem-se diversos problemas de saúde, como a desativação das funções sexual e digestiva, depressão, ansiedade aguda, hipertensão arterial, úlceras, psoríase e diabetes (Alchieri \& Cruz, 2004; Lipp \& Malagris, 2001; Malagris, 2004), assim como abuso de substâncias (Lambert \& Kinsley, 2006).

As contribuições que o presente estudo poderá trazer, portanto, estão ligadas à possibilidade de planejamento de políticas de assistência estudantil que estipulem medidas preventivas e desenvolvam estratégias de manejo, caso seja observada a presença de estresse nos alunos. Quanto mais amplo o conhecimento da realidade dos alunos de pós-graduação, certamente maiores serão as possibilidades de se pensar em políticas de assistência estudantil que, de fato, sejam efetivas para esse grupo de indivíduos.

Esse estudo inseriu-se em um escopo mais amplo de concepção e fundamentação da política de atenção aos alunos de pós-graduação no período de existência da DAE, podendo, portanto, colaborar como embasamento para o planejamento de futuras ações de intervenção junto 
a esse segmento. O objetivo do estudo foi caracterizar uma amostra de estudantes de pós-graduação dos diversos centros da UFRJ e investigar a presença e nível de estresse nela.

\section{Método}

\section{Participantes}

Foram participantes voluntários da pesquisa 140 alunos de pós-graduação da Universidade Federal do Rio de Janeiro (UFRJ), sendo 17 deles pertencentes ao Centro de Ciências da Matemática e da Natureza (CCMN), 20 ao Centro de Letras e Artes (CLA), 20 ao Centro de Filosofia e Ciências Humanas (CFCH), 20 ao Centro de Ciências Jurídicas e Econômicas (CCJE), 20 ao Centro de Ciências da Saúde (CCS), 23 ao Centro de Tecnologia (CT) e 20 ao Fórum de Ciência e Cultura (FCC).

\section{Local}

A pesquisa foi desenvolvida nos programas de Pós-graduação de todos os seis Centros Universitários da UFRJ acima mencionados.

\section{Instrumentos}

Os instrumentos usados se constituíram no Inventário de Sintomas de Estresse para Adultos de Lipp (ISSL), elaborado e padronizado por Lipp (2000) e validado por Lipp e Guevara (1994), em um breve questionário informativo (QI) elaborado pelos autores do estudo, assim como em uma apostila elaborada por Lipp (2000) presente no manual do ISSL (publicada e vendida separadamente), com informações instrutivas sobre estresse e seu manejo. O ISSL, baseado no modelo quadrifásico do estresse (Lipp, 2003), tem como objetivo identificar a presença de estresse, revelar a fase do estresse na qual a pessoa se encontra e a predominância de sintomas físicos e, ou, psicológicos. O ISSL é constituído de três quadros: o primeiro se refere aos sintomas apresentados nas últimas 24 horas (fase de alerta); o segundo é relativo aos sintomas experimentados na última semana (fases de resistência e quase exaustão) e o terceiro é referente aos sintomas apresentados no último mês (fase de exaustão). O breve questionário informativo teve como objetivo conhecer alguns aspectos sociobiográficos dos participantes, tais como idade, sexo, estado civil, naturalidade, filhos, renda mensal, o fato de morar sozinho ou com outras pessoas, nível escolaridade, recebimento ou não de bolsa de estudos, atividades profissionais. 
A apostila oferecida aos estudantes inclui conceitos teóricos sobre estresse, como definição, fases, sintomas, e também recomendações sobre como controlar o estresse. A autora do ISSL sugere que a apostila seja oferecida aos indivíduos que realizam o inventário.

\section{Procedimento}

Primeiramente, por uma tabela aleatória (SAMPLE), foi decidido que a amostra deveria ser composta de 140 alunos. O projeto foi submetido e aprovado pelo Comitê de Ética em Pesquisa da Escola de Enfermagem Anna Nery no dia 13 de fevereiro de 2007. Em seguida, foi apresentada uma carta assinada pelo pró-reitor de Pós-graduação em Pesquisa para as secretarias acadêmicas de todos os centros envolvidos, contendo tanto informações sobre a realização do estudo e seus objetivos quanto sobre o fator motivacional da aplicação do ISSL e do QI.

Com o apoio das secretarias acadêmicas, os alunos foram contatados, respeitando a proximidade e o horário do ambiente de estudo de cada um. Antes da aplicação dos instrumentos, os alunos assinaram um termo de consentimento livre e esclarecido, contendo informações sobre os objetivos e as implicações do estudo.

No final da aplicação, os participantes foram informados pelos investigadores sobre o resultado da avaliação do estresse e receberam a apostila citada acima, no item "instrumentos", explicando o que é o estresse, suas fases, sintomas, assim como estratégias de manejo do estresse, as quais foram discutidas com os participantes.

\section{Resultados}

\section{Análise dos dados}

Os dados obtidos foram analisados por meio de estudo das percentagens referentes às variáveis envolvidas na pesquisa, bem como por estudo e verificação de relações de dependência entre os dados coletados por estatística não paramétrica (teste do qui-quadrado).

Inicialmente, foram analisados os dados referentes ao questionário informativo; em seguida, os dados do ISSL e, finalmente, foram consideradas as relações de dependência entre os dados dos dois instrumentos. Dessa forma, os resultados e sua análise serão apresentados a seguir.

\section{Questionário informativo - QI}

Os dados coletados por meio do questionário informativo possibilitaram caracterizar a amostra. Observou-se que a idade média dos participantes era de 
$30.3 \pm 7.5$, variando de 21 a 59 anos. Quanto ao sexo, verificou-se que 74 (53\%) eram mulheres, e 66 (47\%) eram homens; e quanto ao estado civil, 91 (65\%) eram solteiros, e 38 (27\%) casados ou vivendo com um companheiro. Quanto à naturalidade, 87 (62,1\%) dos participantes eram do Rio de Janeiro, $30(21 \%)$ de outros estados do Sudeste e os demais se dividiam entre outros estados do Brasil e o exterior, sendo 138 (98,6\%) brasileiros e apenas 2 $(1,4 \%)$ estrangeiros. Entre os participantes, 48 (34,5\%) residiam no Rio de Janeiro especificamente para cursar a pós-graduação. Quanto à residência, 50 $(35,7 \%)$ moravam com os pais, $47(33,6 \%)$ com outros parentes, $24(17,1 \%)$ com amigos em república/casa de estudantes ou pensionato e 19 (13,6\%) moravam sozinhos.

Verificou-se que $110(79,1 \%)$ não tinham filhos, e os demais tinham de 1 a 3 filhos. Quanto à renda mensal, observou-se que 34 (25\%) recebiam de 1 a 5 salários mínimos, e 103 (75\%) recebiam acima de 5 .

Pôde-se verificar que 75 (54\%) faziam mestrado, e 65 (46\%) doutorado. Dos alunos de mestrado, $42(56 \%)$ estavam cursando o primeiro período, 3 (4\%) o segundo período, $14(18,6 \%)$ o terceiro e a mesma porcentagem o quarto período. Entre os alunos de doutorado, 16 (24,6\%) estavam no primeiro período, $5(7,7 \%)$ no segundo, $13(20 \%)$ no terceiro, $3(4,6 \%)$ no quarto período, $7(10,8 \%)$ no quinto período, $4(6,1 \%)$ no sexto, 8 $(12,3 \%)$ no sétimo e $5(7,7 \%)$ cursavam o oitavo período. Quanto à bolsa de estudos, verificou-se que $59(42,4 \%)$ não a tinham, e, daqueles que a tinham (57,6\%), $38(27,3 \%)$ a recebiam da CAPES, $31(22,3 \%)$ do CNPq, $3(2,2 \%)$ da FAPERJ e $8(5,8 \%)$ de outras instituições. Verificou-se que $66(47 \%)$ dos estudantes tinham outras atividades profissionais além de cursarem a pósgraduação e 74 (52\%) não. Convém enfatizar que alguns participantes não responderam a alguns itens do questionário, por isso adaptou-se o cálculo das porcentagens.

\section{Inventário de sintomas de estresse para adultos de Lipp - ISSL}

No que se refere aos resultados obtidos no ISSL, verificou-se que 82 $(58,6 \%)$ estavam eestresseados na época da avaliação. Considerando o curso, verificou-se que, do total de 75 alunos de mestrado e 65 de doutorado, 42 (56\%) e $40(62 \%)$ estavam eestresseados (tabela 1).

Observou-se que $50 \%$ dos estudantes estavam na segunda fase do estresse (resistência), 6,4\% estavam na terceira fase do estresse (quase exaustão), $1,4 \%$ na fase de exaustão ( $4^{\mathrm{a}}$ fase) e $0,7 \%$ na primeira fase (alerta) (tabela 1 ). Quanto ao tipo de sintoma de estresse predominante, observou-se que 74,4\% 
apresentavam mais sintomas psicológicos como manifestação do estresse, $17,1 \%$ apresentavam predominância de sintomas físicos e 8,5\% apresentavam igualmente ambos os tipos de sintomas.

Tabela 1

Presença e fase de estresse de acordo com o curso

\begin{tabular}{|c|c|c|c|c|c|c|c|c|c|c|c|c|c|}
\hline Curso & $\begin{array}{l}\text { Sem } \\
\text { Estresse }\end{array}$ & & A & & $\mathbf{R}$ & & $\mathrm{QE}$ & & $\mathbf{E}$ & & $\begin{array}{l}\text { Total } \\
\text { Estresse }\end{array}$ & & Total \\
\hline & $\mathrm{F}$ & $\%$ & $F$ & $\%$ & $\mathrm{~F}$ & $\%$ & $\mathrm{~F}$ & $\%$ & $\mathrm{~F}$ & $\%$ & $F$ & $\%$ & $\mathrm{~F}$ \\
\hline $\begin{array}{l}\text { Mestrado } \\
\text { (75) }\end{array}$ & 33 & 44 & 0 & 0 & 35 & 83.3 & 5 & 12 & 2 & 4.7 & 42 & 56 & 75 \\
\hline $\begin{array}{l}\text { Douto- } \\
\text { rado } \\
(65)\end{array}$ & 25 & 38.4 & 1 & 2.5 & 35 & 87.5 & 4 & 10 & 0 & 0 & 40 & 62 & 65 \\
\hline Total & 58 & 41.4 & 1 & 0.7 & 70 & 50 & 9 & 6.4 & 2 & 1.4 & 82 & 58.6 & 140 \\
\hline
\end{tabular}

Nota: $\mathrm{A}$ = Alerta. $\mathrm{R}=$ Resistência. QE = Quase-Exaustão. $\mathrm{E}$ = Exaustão.

Levando-se em conta a relação entre o índice de estresse o centro universitário e o total avaliado em cada centro, verificou-se que o CCMN apresentou o maior índice de eestresseados entre seus alunos $(82,4 \%)$, seguido pelo CT com $61 \%$, o FCC com $60 \%$, o CLA e o CFCH com $55 \%$ e, por último, o CCJE e CCS com 50\% (tabela 2). A análise estatística por meio do teste qui-quadrado revelou não haver diferença significativa entre o índice de eestresseados dos diversos centros estudados $(\mathrm{p}=0,4875)$.

\section{Tabela 2}

Índice de estresse de acordo com o Centro Universitário

\begin{tabular}{ccccccc}
\hline Centro & $\begin{array}{l}\text { Sem Es- } \\
\text { tresse }\end{array}$ & \multicolumn{3}{c}{ Com Estresse } & \multicolumn{2}{c}{ Total } \\
\hline & $\mathrm{F}$ & $\%$ & $\mathrm{~F}$ & $\%$ & $\mathrm{~F}$ & $\%$ \\
\hline $\mathrm{CCMN}$ & 3 & 17,5 & 14 & 82,4 & 17 & 100 \\
\hline $\mathrm{CT}$ & 9 & 39 & 14 & 61 & 23 & 100 \\
\hline $\mathrm{CCJE}$ & 10 & 50 & 10 & 50 & 20 & 100 \\
\hline $\mathrm{CLA}$ & 9 & 45 & 11 & 55 & 20 & 100 \\
\hline $\mathrm{CFCH}$ & 9 & 45 & 11 & 55 & 20 & 100 \\
\hline $\mathrm{CCS}$ & 10 & 50 & 10 & 50 & 20 & 100 \\
\hline $\mathrm{FCC}$ & 8 & 40 & 12 & 60 & 20 & 100 \\
\hline
\end{tabular}

Nota: O CCMN e CT tiveram número diferente de estudantes por problemas ocorridos na aplicação. 


\section{QI e ISSL}

Pelo teste do qui-quadrado, procurou-se investigar possíveis relaçôes de dependência entre as variáveis do questionário informativo e o resultado obtido no ISSL. As seguintes variáveis do questionário informativo foram relacionadas ao estresse: sexo, nível da pós-graduação (mestrado e doutorado), estado civil, o fato de ter ou não bolsa de estudo, de ter ou não filhos e ter ou não outras atividades além da pós-graduação. A análise estatística revelou que somente o sexo e o fato de ter ou não outra atividade além da pós-graduação têm relação com o estresse, encontrando diferença significativa $(\mathrm{p}<0,05)$. Na amostra estudada, havia mais mulheres eestresseadas $(67,6 \%)$ do que homens eestresseados $(48,4 \%)$, e os alunos que não tinham outras atividades fora da pós estavam mais eestresseados $(67,6 \%)$ do que os que tinham $(48,4 \%)$.

\section{Tabela 3}

Relações entre dados do QI e do ISSL

\begin{tabular}{llll}
\hline Variáveis QI & Sem estresse & Com estresse & p \\
\hline Sexo feminino & 34 & 32 & $0,02^{*}$ \\
Sexo masculino & 24 & 50 & 0,59 \\
\hline Até 30 anos & 36 & 55 & 0,16 \\
Acima de 30 anos & 22 & 27 & 0,56 \\
\hline Possui bolsa & 29 & 30 & \\
Não possui bolsa & 29 & 51 & 0,2 \\
\hline Solteiro & 40 & 61 & \\
Casado ou com companheiro & 18 & 21 & 0,6 \\
\hline Sem filhos & 42 & 68 & \\
Com filhos & 15 & 42 & $0,02^{*}$ \\
\hline Mestrado & 33 & 40 & \\
Doutorado & 25 & 32 & 50 \\
\hline Possui atividades fora da pós & 34 & & \\
Não possui atividades fora da pós & 24 & & \\
\hline
\end{tabular}

${ }^{*} \mathrm{P}<0.05$

\section{Discussão}

No que se refere à caracterização da amostra estudada, os resultados encontrados nesta pesquisa mostraram que os alunos da Pós-graduação da 
UFRJ investigados são relativamente jovens, já que a média de idade foi de 30 anos, e que há uma pequena predominância de mulheres $(53 \%) \mathrm{em}$ relação aos homens (47\%). A relativa pouca idade dos participantes pode estar relacionada ao fato de os estudantes estarem buscando a pós-graduação poucos anos após terminarem a graduação ou até mesmo imediatamente após o término da faculdade. Talvez a grande competitividade e a escassez de oportunidades no mercado de trabalho estejam contribuindo para essa busca. Um outro motivo pode ser também a necessidade da bolsa de estudos para complementar a renda, devido à falta de colocação no mercado de trabalho assim que se formam.

Além disso, alguns estudantes se envolvem na área de pesquisa ainda na graduação. Sendo assim, quando se formam, já há um interesse em dar continuidade à atividade científica.

É importante lembrar que, no estudo brasileiro de Santos e Alves Junior (2007) relativo ao segmento da pós-graduação, a faixa etária predominante era entre 30 e 39 anos, o que se mostra um pouco acima da realidade aqui encontrada. É possível que a faixa etária de alunos nos programas de pósgraduação, varie um pouco de acordo com a região do Brasil por causa da cultura e das oportunidades locais. O estudo de Santos e Alves Junior foi realizado em Sergipe, e este estudo no Rio de Janeiro.

A questão de a amostra ser praticamente proporcional no que se refere ao sexo mostra o quanto as mulheres têm se tornado cada vez mais ativas na busca por qualificação e competitividade. $\mathrm{O}$ resultado pode indicar que o papel da mulher na sociedade ao longo dos anos tem se ampliado da atividade profissional para o desenvolvimento acadêmico através do ingresso na pósgraduação.

A maioria da amostra ser composta de alunos solteiros (65\%) parece ter relação com o fato de os alunos serem jovens e talvez não estarem empregados, pois foi verificado que $52 \%$ não tinham outra ocupação além da pós-graduação e 35,7\% ainda moravam com os pais. A mesma explicação parece razoável para o fato de $79,1 \%$ não terem filhos.

Quanto à naturalidade, verificou-se que 62\% eram do Rio de Janeiro e apenas $1,4 \%$ do exterior. Esse dado revela o quanto a pós-graduação da UFRJ é regionalizada, demonstrando, assim, a necessidade de maior divulgação dos cursos oferecidos em outros estados do Brasil e no exterior, de modo a haver uma maior troca entre saberes de diferentes regiōes do Brasil e do mundo. É possível que outras universidades brasileiras compartilhem da mesma realidade, fato que merece maior investigação. 
Especificamente quanto a questões acadêmicas, considerando o curso de mestrado, $56 \%$ estavam ainda no primeiro período. No curso de doutorado, observou-se que $24,6 \%$ estavam no primeiro período. Tal dado leva a pensar que grande parte da amostra ainda se encontrava em período de adaptação, cursando as disciplinas. Esse período requer a aprendizagem de uma série de regras específicas da pós-graduação, horários rígidos e a realização de avaliações nas disciplinas, o que pode ser bastante eestresseante para os alunos. No entanto, os $18,6 \%$ dos alunos do mestrado e os $7,7 \%$ do doutorado que estavam no último período também sofriam pressões relacionadas à defesa e aos prazos a serem cumpridos.

Verificou-se que 57,6\% da amostra de estudantes recebiam bolsa de estudo, sendo que entre as bolsas concedidas, a CAPES (27,3\%) foi a instituição com a maior oferta, seguida do CNPq (22,3\%). Esses números revelam ainda a carência dos programas no recebimento de bolsas, já que um grande número de alunos não as tem. Tal dado demonstra a necessidade da implementação de políticas para buscar a ampliação de concessão de bolsas, de modo que o aproveitamento do aluno possa ser maior, já que, com bolsa, ele terá maior disponibilidade de tempo para dedicar-se ao estudo. O fato de 52,9\% dos alunos terem outras atividades profissionais além da pós-graduação parece ser reflexo da carência de auxílio de bolsas de estudo.

Quanto à presença de estresse entre os alunos investigados, verificouse que 58,6\% estavam eestresseados, índice que está acima do divulgado em pesquisas realizadas no Brasil sobre o índice de estresse na população (Lipp \& Malagris, 2004), que gira em torno de 35\%. No entanto, estudos anteriormente aqui citados encontraram um índice de 40,7\% (Santos \& Alves Júnior, 2007) em estudantes brasileiros de pós-graduação e $66,4 \%$ em estudantes americanos (Pfeifer, Kranz \& Scoggin, 2008). Logo, de acordo com a literatura, os índices de estresse encontrados entre alunos de pósgraduação têm se mostrado altos e preocupantes no Brasil e no exterior.

Pode-se supor que as demandas da pós-graduação em termos de prazos, número de horas de estudo (que, em alguns casos, se somam às horas em laboratório de pesquisa), relacionamento interpessoal com orientadores, professores e colegas podem constituir-se em fontes de estresse, como afirmam De Méis, Velloso, Lannes, Carmo e De Méis (2003) ao se referirem ao desgaste emocional sofrido pelas pessoas envolvidas no desenvolvimento científico. Somadas a essas, pode-se pensar em características individuais dos estudantes, como a falta de maturidade para cursar a pós-graduação, já que a amostra aqui estudada é de maioria jovem. Tais características 
podem gerar interpretações disfuncionais e atitudes desadaptativas que contribuam para o desenvolvimento do estresse, como enfatiza Straub (2005) ao referir-se à importância da avaliação cognitiva e dos recursos de enfrentamento do indivíduo.

Mostram-se necessários estudos que procurem investigar as fontes de estresse desses estudantes, para que sejam propostas mudanças na pós-graduação, de modo a contribuir para a redução nos índices de estresse. Além disso, fazse necessária a implementação de programas de controle de estresse para os alunos. Tal sugestão é coerente com os estudos de Alchieri e Cruz (2004) e de Lambert e Kinsley (2006) quanto à prevenção de problemas de saúde através do controle do estresse.

É também importante enfatizar que a fase de estresse prevalente entre os estudantes foi a de resistência (50\%). Tal se constitui na segunda fase do estresse, na qual o organismo está esforçando-se para recuperar-se do desgaste sofrido no enfrentamento de eestresseores. Nessa fase, o indivíduo sente cansaço, desgaste, perda da libido, dificuldade de memória e concentração, hipersensibilidade emotiva, dúvidas quanto a si próprio, além de outros sintomas (Lipp \& Malagris, 2001). É fácil imaginar os prejuízos que esses alunos podem ter por estarem nessa fase do estresse, pois a demanda (prazos e exigências) é grande, e o organismo está debilitado para atendê-la. Nesses casos, existe a possibilidade de os alunos passarem para fases mais avançadas do estresse e virem a apresentar problemas de saúde física ou psicológica. No que se refere aos sintomas de estresse predominantes na amostra, os sintomas psicológicos foram os prevalentes, incluindo preocupação, ansiedade, tensão, entre outros. Tais sintomas podem perturbar bastante o desempenho acadêmico e a qualidade de vida do estudante. Não se pode deixar de notar que 9 alunos estavam na fase de quase exaustão e 1 na de exaustão, que são fases do estresse altamente preocupantes, já que, na fase de quase exaustão, os problemas de saúde se iniciam e, na de exaustão, eles se estabelecem. Os resultados aqui encontrados quanto à fase de estresse predominante estão de acordo com o estudo de Santos e Alves Junior (2007), no qual 81,8\% dos estudantes estavam na fase de resistência. No entanto, houve diferença em relação aos sintomas, já que, neste estudo, predominaram sintomas psicológicos $(74,4 \%)$ e, no dos autores citados, sintomas físicos $(63,6 \%)$. Seria interessante realizar estudos comparativos entre regióes do Brasil para investigação sobre o modo de manifestação do estresse.

Foi interessante também observar o índice de estresse de acordo com o centro universitário, calculado com base no total de alunos de cada centro. 
Verificou-se que o CCMN apresentou o maior índice de estresse entre seus alunos, com um total de $82,4 \%$. Em seguida, vem o CT, com $61 \%$; o FCC, com 60\%; o CLA e o CFCH, com 55\%; o CCJE e o CCS, com 50\%. Como a pesquisa não teve como objetivo analisar os eestresseores presentes, não é possível explicar as razóes pelas quais essa ordem foi encontrada. Uma série de questôes pode ser levantada; no entanto, sugere-se novo estudo voltado para a investigação dos eestresseores de cada centro universitário. Os resultados indicam a importância de verificarem-se as políticas acadêmicas internas, regras, prazos, relação do aluno com o orientador, ambiente físico e psicossocial. Como já citado neste artigo, Louzada e Silva Filho (2005) enfatizam que a vulnerabilidade dos pós-graduandos ao estresse relacionase a tais questões; logo, parece relevante investigar o segmento em questão para a confirmação dessas afirmações.

Considerando os dados do QI e o ISSL, várias relaçôes foram estudadas, encontrando-se dependência entre estresse e sexo, havendo mais mulheres eestresseadas. Tal dado é coerente com vários estudos que incluem a relação entre o gênero e o fator estresse. $\mathrm{Na}$ maioria das vezes, esse tipo de investigação revela um maior índice de mulheres eestresseadas (Lipp \& Malagris, 2004; Santos e Alves Junior, 2007). Uma hipótese a ser pensada é o fato de que a mulher acumula diversos papéis no seu dia a dia. Além disso, em alguns contextos, ela sofre discriminação e, por isso, encontra mais obstáculos para seu desempenho. Não se pode afirmar que exista discriminação no contexto estudado, mas é uma hipótese que poderia ser investigada. Também foi observada a relação de dependência entre estresse e o fato de o aluno ter ou não atividade profissional além da pós-graduação. Nessa última relação estudada, verificou-se predominância de estresse entre aqueles que não têm outra atividade. É possível que aqueles que se ocupam de uma atividade profissional tenham maior condição financeira que possibilite melhor qualidade de vida. É provável que eles tenham satisfação por estarem inseridos no mercado de trabalho e, com isso, sintamse mais seguros quanto ao futuro. A preocupação financeira foi um dos eestresseores enfatizados também na literatura internacional por meio dos estudos de Byars (2005) e Hyun, Quinn, Madon e Lustig (2007) referentes a alunos de pós-graduação. Ao refletir sobre o resultado aqui encontrado, é interessante observar que o fato de ter bolsa de estudos não se revela um fator redutor de estresse, enquanto que ter atividade profissional sim. É possível que a bolsa, embora ajude o aluno durante o curso, não chegue a tranquilizá-lo quanto ao futuro financeiro e profissional, ao contrário da atividade profissional. 


\section{Conclusão}

Este estudo demonstra uma preocupação com a qualidade de vida dos estudantes, e os resultados revelaram que essa preocupação procede, pois se encontrou um alto índice de estresse entre os estudantes. $\mathrm{O}$ fato de a maioria dos alunos situar-se na segunda fase do estresse acentua a preocupação, devido aos possíveis prejuízos na vida acadêmica e pessoal deles. O fato de haverem mais mulheres eestresseadas é outro dado a ser aprofundado para que se possa compreender o porquê desse fato. O que estaria levando esses alunos a estarem eestresseados? Por que existe um índice de estresse mais elevado entre aqueles que não têm ocupação profissional além da pós-graduação? Por que alguns centros universitários têm índice de estresse mais elevado do que outros? De que forma é possível ajudar esses alunos? Serão necessárias mudanças estruturais na pós-graduação? Será que o estresse é esperado entre alunos que estejam cursando pós-graduação? É necessário realizar pesquisas, de modo a encontrar possíveis respostas a essas perguntas e pensar em soluçôes. É fundamental que políticas de mudança ou de ajuda à saúde física e psicológica dos alunos sejam elaboradas e implantadas. Este estudo pode constituir-se em uma alavanca para outras investigações e ações.

\section{Referências}

Ahmad, A. \& Bano, M. (2008). Academic stress among postgraduate students before and after announcement of result. Gyanodaya, 1 (1). Recuperado em 26 de fevereiro, 2009 de http://www.IndianJournals.com.

Alchieri, J. C. \& Cruz, R. M. (2004). Eestressee: conceitos, métodos, medidas e possibilidades de intervenção. São Paulo: Casa do Psicólogo.

Baptista, M. N. \& Campos, L. F. L. (2000). Avaliação longitudinal de sintomas de depressão e Estresse em estudantes de Psicologia. Boletim de Psicologia, 50 (113), 37-58.

Bujdoso, Y. L. V. (2005). Dissertação como eestresseor: em busca de seu significado para o mestrando de enfermagem. Dissertação de mestrado não publicada, Universidade de São Paulo, São Paulo.

Calais, S. L.; Carrara, K.; Brum, M. M. et. al. (2007). Estresse entre calouros e veteranos de Jornalismo. Estudos de Psicologia, 24 (1), 69-77.

Cannon, W. B. (1939). The wisdom of the body. Nova Iorque: Norton. 
Csef, H. \& Hefner J. (2005). Stress and myocardial infarction. MMW Fortschr Med., 147 (13), 33-35.

De Meis, L.; Velloso, A.; Lannes, D.; Carmo, M. S. \& De Meis, C. (2003). The growing competition in Brazilian science: rite of passage, stress and burnout. Brazilian Journal of Medical and Biological Research, 36 (9), 1135-1141.

Duque, J. C.; Brondani, J. T. \& Luna, S. P. L. (2005). Estresse e pós-graduação em Medicina Veterinária. Revista Brasileira de Pós-Graduação, 2 (3), 134-148.

Gazzaniga, M. S. \& Heatherton, T. F. (2005). Ciência Psicológica: mente, cérebro e comportamento. Porto Alegre: Artmed.

Hyun, J.; Quinn, B.; Madon, T. \& Lustig, S. (2007). Mental health need, awareness, and use of counseling services among international graduate students. Journal of American College Health, 56 (2), 109-118.

Greenberger, D. \& Padesky, C. A. (1999). A mente vencendo o humor. Porto Alegre: Artmed.

Lambert, K. \& Kinsley, C. H. (2006). Neurociência clinica: as bases neurobiológicas da saúde mental. Porto Alegre: Artmed.

Lipp, M. E. N. (1996). Estresse: conceitos básicos. In: Lipp, M. E. N. Pesquisas sobre estresse no Brasil: saúde, ocupações e grupos de risco. (p. 17-31). Campinas: Papirus.

Lipp, M. E. N. (2000). Manual do inventário de sintomas de estresse para adultos de Lipp. São Paulo: Casa do Psicólogo.

Lipp, M. E. N. (2003). O modelo quadrifásico do stress. In: M. E. N. Lipp (org.) Mecanismos neuropsicofisiológicos do estresse: teoria e aplicaçôes clínicas. (p. 17-21). São Paulo: Casa do Psicólogo.

Lipp, M. E. N. (2004a). O que eu tenho é stress? De onde ele vem? In: M. E. N. Lipp (org.). O estresse está dentro de você. (p. 11-18). São Paulo: Contexto.

Lipp, M. E. N. (2004b). Estresse emocional: esboço da teoria de "temas de vida”. In: M. E. N. Lipp (org.). O stress no Brasil: pesquisas avançadas. (p. 17-30). Campinas: Papirus.

Lipp, M. E. N. (2005). Estresse e o turbilhão da raiva. São Paulo: Casa do Psicólogo. 
Lipp, M. E. N. et. al. (1991). Estudo experimental de duas condiçōes de tratamento médico-psicológico a pessoas portadoras de psoríase. Campinas: Unicamp, NEP.

Lipp, M. E. N. \& Novaes, L. E. (2003). Conhecer \& enfrentar o estresse. São Paulo: Contexto.

Lipp, M. E. N. \& Malagris, L. E. N. (2001). O estresse emocional e seu tratamento. In: B. P. Range (org.) Psicoterapias cognitivo-comportamentais: um diálogo com a psiquiatria. (p. 475-490). Porto Alegre: Artmed.

Lipp, M. E. N. \& Guevara, A. J. H. (1994). Validação empírica do inventário de sintomas de estresse. Estudos de Psicologia, 11 (3), 43-49.

Lipp, M. E. N. \& Malagris, L. E. N. (1995). Manejo do eestressee. In: B. P. Range (org.). Psicoterapia Comportamental e Cognitiva: pesquisa, prática, aplicaçôes e problemas. (p. 215-222). Campinas: Livro Pleno.

Lipp, M. E. N. \& Malagris, L. E. N. (2004). O estresse no Brasil de hoje. In: M. E. N. Lipp (org.). O estresse no Brasil: pesquisas avançadas. (p. 215-222). Campinas: Papirus.

Lipp, M. E. N.; Malagris, L. E. N. \& Novais, L. E. (2007). Estresse ao longo da vida. São Paulo: Ícone.

Louzada, R. C. R. \& Silva Filho, J. F. (2002). Sofrimento psíquico e formação de pesquisadores. Revista Brasileira de Psiquiatria, 24 (2), 120-141.

Louzada, R. C. R. \& Silva Filho, J. F. (2005). Formação do pesquisador e sofrimento mental: um estudo de caso. Psicologia em Estudo, 10 (3), 451461.

Malhotra S. K. \& Mehta V. (2008). Role of estresseful life events in induction or exacerbation of psoriasis and chronic urticaria. Indian Journal of Dermatology, Venereology and Leprology, 74 (6), 594-559.

Malagris, L. E. N. (1996). Estresse e úlceras gastroduodenais: interações clínico-psicológicas. In: Lipp, M. (org.). Pesquisas sobre estresse no Brasil: saúde, ocupações e grupos de risco. (p. 83-108). São Paulo: Papirus.

Malagris, L. E. N. (2004). A via l-arginina-óxido nitrico e o controle do estresse em pacientes com hipertensão arterial sistêmica. Tese de Doutorado, Programa de Fisiopatologia Geral e Experimental, Universidade do Estado do Rio de Janeiro, Rio de Janeiro. 
Malagris, L. E. N. \& Fiorito, A. C. C. (2006). Avaliação do nível de estresse de técnicos da área de saúde. Estudos de Psicologia, 23 (4), 391-398.

Margis, R., Picon, P., Cosner, A. F. et. al. (2003). Relação entre eestresseores, eestressee e ansiedade. Revista de Psiquiatria do Rio Grande do Sul, 25 (1), 6574.

Nogueira-Martins, L. A.; Fagnani Neto, R.; Macedo, P. C. M.; Cítero, V. A. \& Mari, J. J. (2004). The mental health of graduate students at the Federal University of São Paulo: a preliminary report. Brazilian Journal of Medical and Biological Research, 37 (10), 1519-1524.

Pfeifer, T. A.; Kranz, P. L. \& Scoggin, A. E. (2008). Perceived stress in occupational therapy students. Occupational Therapy International. 15 (4), 221-231.

Pinto, W. N. R. (1996). Estresse, qualidade de vida e vitiligo. In: M. E. N. Lipp (org.). Pesquisas sobre estresse no Brasil: saúde, ocupação e grupos de risco. (p. 129-148). Campinas: Papirus.

Schmitt, L. H. (1999). The assessment of stress in traditional societies. In: A. Bittles \& P. A. Parsons (eds.). Estresse: evolutionary, biosocial and clinical perspectives. (p. 81-99). Londres: The Galton Institute.

Praag, H. M., Kloet, R. \& Os, J. (2005). Stress, cérebro e depressão. Lisboa: Climepsi.

Santos, A. F. \& Alves Junior, A. (2007). Eestressee e estratégias de enfrentamento em mestrandos de ciências da saúde. Psicologia: Reflexão e Crítica, 2007, 20 (1), 104-113. Disponível em: <http://www.scielo.br/scielo.php?script=sci_ arttext\&pid=S0102-79722007000100014\&lng=en\&nrm=iso >. (Acesso em: 25 fev. 2009).

Selye, H.(1965). Stress: a tensão da vida. São Paulo: Ibrasa.

Stecker T. (2004). Well-being in an academic environment. Medical Education, 38 (5), 465-78.

Straub, R. O. (2005). Psicologia da saúde. Porto Alegre: Artmed. 